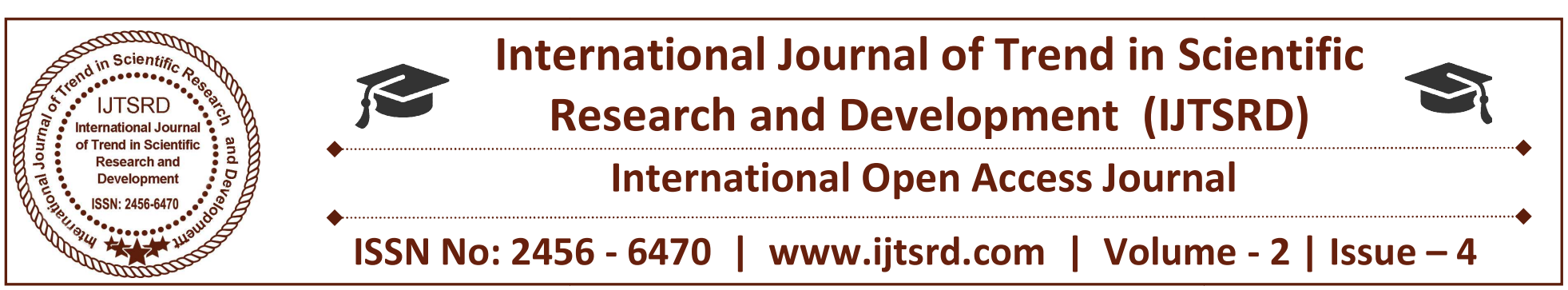

\title{
Multifactor Based Top K Feature Extraction Using Summarized Customer Reviews
}

\author{
Dr. Saravana Balaji B ${ }^{1}$, ShruthiShree S. $H^{2}$, Adarsh $A^{3}$, Akshatha S Kumar ${ }^{4}$, Pranav P. $M^{5}$ \\ ${ }^{1}$ Associate Professor, ${ }^{2}$ Assistant Professor, ${ }^{3,4,5}$ B.Tech Final Year Students \\ $1,2,3,4,5$ Department of Information Science and Engineering \\ ${ }^{1,2,3,4,5}$ School of Engineering and Technology - Jain University, Bangalore, Karnataka, India
}

\begin{abstract}
It is a typical practice that vendors offering items on the Web request that their clients review the item. These remarks are essential for potential clients when choosing which item to purchase. As internet business is increasingly well known, the quantity of client surveys that an item gets develops quickly. In any case, perusing a lot of client surveys accessible for every item is a tedious procedure. Hence, clients generally tend to peruse little bits of highest remarks and avoid whatever remains of them. For an item, the quantity of audits can be in hundreds or thousands.
\end{abstract}

In this task, our principle objective is to abridge all the client surveys of an item. This diagram task isn't the indistinguishable customary substance abstract since we are simply enthused about the specific features of the thing that customers have evaluations on and moreover whether the conclusions are certain or negative. We outline the reviews of an item class by producing the sentiment score for each survey and after that summarize all the opinion scores from each review.

Keywords: Sentiment Analysis, Natural Language Processing, Feature Based Opinion Mining, Review Summarization, Information Extraction.

\section{INTRODUCTION}

With the developing fame of web, web based business sites are taking an ever-increasing number of spots in our lives. These days, a developing number of individuals are shopping on the web. Using the product review feature of e-commerce web sites, these

customers are submitting remarks and proclaiming their conclusions about the products as well as indicating fulfillment with the items. These item surveys are the essential purpose behind the expanding quantities of web based shopping clients since they importantly affect the choice of the clients' which item to pick. The item review help customers to choose the best item that addresses their issues. Utilizing the item audit highlight, every client can post diverse remarks about various determinations of an item. In any case, the surveys mirror the individual judgments of the clients on the grounds that their necessities and the desires may contrast from numerous points of view. Subsequently, a survey is absolutely subjective and it gives vital individual criticism about the item. Additionally, more often than not clients don't want to peruse each and every item audit that is accessible. Now, synopsis procedures turn out to be extremely helpful in demonstrating the general thought of the surveys. To get the attributes of client conduct, the audits ought to be nostalgically broke down so as to decide the positive/negative sides of the item.

In this universe of overpowering number of brands, item audits assume an essential part in obtaining choice. Basically, item audits are fundamental for both the two purchasers and dealers. Each organization advocates their item is the best. But in reality, it's the consumer who can decide which product is better by using it. Knowing the advantages and disadvantages of a specific item or administration from the general population who have encountered in direct, enables you to settle on educated choice for 
your buy. There are numerous sites that give item surveys including the shopping sites like Flipkart and amazon. Those sites for the most part have a rating, advantages and disadvantages segment for each audit. Shoppers generally go through these reviews before choosing what to purchase.

Product reviewing is a device that organizations can use to influence their item or administration to emerge in the market. It additionally encourages them to examine what the clients expect in the up and coming variants of the item.

The encounters and conclusions from different clients can contribute data about the quality and estimation of an item and can accordingly lessen client's decision hazard and supplement different types of business-toclient correspondence. To get data about the item, a client can look audits on the web. Be that as it may, gathering and investigating surveys from numerous sources about chosen items brings an issue. The volume of the information is colossal, and individuals can't process them physically inside an adequate time. PCs can't comprehend content written in characteristic dialect and can't understand the assessment or uncover the slant of surveys. Regardless of these challenges, it is conceivable and in circumstances there are a considerable measure of surveys for a specific item to utilize PCs for examining and revealing the concealed learning. At that point it could be introduced to a client in a particular shape, helping the clients to comprehend and make a move.

In this paper, we propose to ponder the issue of feature based sentiment rundown of client audits of items sold on the web. The undertaking is performed by distinguishing the features of the item that clients have communicated their suppositions on (called opinion features) and rank the features as per their frequencies that they show up in the surveys. With such a component based conclusion outline, a potential client can without much of a stretch perceive how the current clients feel about a specific feature of an item. In the event that the client is especially keen on that feature, at that point he/she can depend upon the gave surveys to settling on a choice about that item.

\section{RELATED WORK}

Our work is primarily identified with two zones of research, one is to outline the content (client audits) and other errand is to locate the best most features on which clients have remarked the most.
Our strategy for content synopsis isn't like traditional text summarization where a large portion of them give the audit report by rewording the content, yet it fluctuates in various ways:

We provide overall score for ' $n$ ' reviews which tells the product reviews are positive or negative.

We just focus on the highlights of a specific item that clients have conclusions on. We don't abridge the surveys by modifying a subset of the first sentences from the audits to catch their fundamental focuses as in the customary content outline.

\section{THE PROPOSED TECHNIQUES}

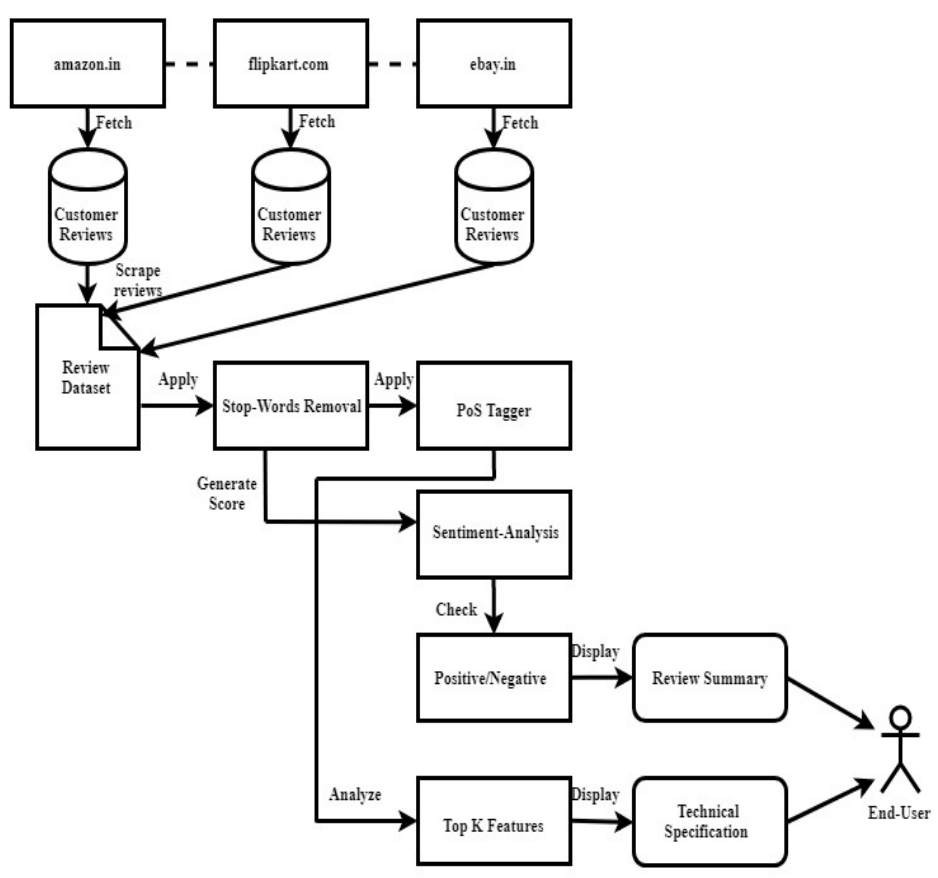

\section{Figure 1: Architecture Diagram}

Figure 1 gives an architectural overview for our review mining and opinion based summarization system. Reviews from various e-commerce websites will be fetched at the initial stage, then for each product review dataset Stop Word algorithm will be applied. Once the Stop Words are eliminated, Parts of Speech Tagger algorithm will be applied to the result which tags each token as Noun / Verb / Adjective / Proverb etc. From this result, we then found the top $\mathrm{k}$ features of the product on which customers have reviewed the products. In this project we provide summarized reviews with a sentiment score by taking the sum of all positive and negative words from the reviews. 


\section{Stop Word removal}

The way toward changing over information to something a PC can comprehend is alluded to as prehandling. One of the critical sorts of pre-dealing with is to filter through vain data. In regular dialect preparing, futile words (information), are alluded to as stop words.

Stop Words: A stop word is a generally utilized word, (for example, "the", "an", "an", "in") that a web index has been customized to overlook, both when ordering sections for seeking and while recovering them as the consequence of a hunt question.

We would not need these words consuming up room in our database, or taking up profitable handling time. For this, we can expel them effectively, by putting away a rundown of words that you consider to be stop words.

\begin{tabular}{|c|c|}
\hline $\begin{array}{c}\text { Sample text with Stop } \\
\text { Words }\end{array}$ & Without Stop Words \\
\hline $\begin{array}{c}\text { GeeksforGeeks - A Computer } \\
\text { Science Portal for Geeks }\end{array}$ & $\begin{array}{c}\text { GeeksforGeeks, Computer Science, } \\
\text { Portal ,Geeks }\end{array}$ \\
\hline Can listening be exhausting? & Listening, Exhausting \\
\hline I like reading, so I read & Like, Reading, read \\
\hline
\end{tabular}

Table 1: Example for Stop words removal
Table 2: Below table represents the PoS tags

\begin{tabular}{|l|l|}
\hline NN & Noun \\
\hline NNS & Noun \\
\hline VBP & Verb \\
\hline JJ & Adjective \\
\hline IN & Preposition \\
\hline DT & Determiner \\
\hline CC & Conjunction \\
\hline
\end{tabular}

\section{Sentiment analysis}

The procedure of computationally distinguishing and arranging assessments communicated in a bit of content, particularly so as to decide if the author's state of mind towards a specific subject, item, and so forth is sure, negative, or impartial.

Example: The picture and sound quality of this TV is excellent.

Here the sentiment of the customer is positive.

\section{Positive/Negative words}

In NLP, it's very important to find the number of positive and negative words in a sentence as it helps to identify the opinion of a user.

Example: Good - Positive, Bad - Negative

\section{Feature extraction or Opinion mining}

It's a process of extracting the opinions of a customer from the reviews. In our paper, top $\mathrm{k}$ features for each product is been extracted to find the overall opinion of a user.

Example: If the customer is reviewing a cell phone and writes a review about camera as "Camera is very poor", his opinion about the phone's camera is negative and the feature he has mentioned is camera.

\section{Experiment Results and Discussion}

Formula to calculate the average score of whole review file of a product: 
Average rating:

\section{If positive words are more than negative words}

$\sum$ Sun $/\left(\sum\right.$ (Positive words) $-\sum$ (Negative words $\left.)\right)$

\section{If negative words are more than positive words}

$\sum$ Sum $/\left(\sum\right.$ (Negative words $)-\sum$ (Positive words $\left.)\right)$

\section{$\sum \quad$ Sum $/\left(\sum\right.$ (Negativewords) - \\ $\sum$ (Positivewords))}

Sum=Total number of words after removing stop words

Positive words $=$ Total count of positive words in the review file, after removing stop words

Negative words $=$ Total count of negative words in the review file, after removing stop words

Table 3: Sentimental score for a sample data

\begin{tabular}{|l|l|l|l|}
\hline $\begin{array}{l}\text { Sum } \\
\text { (Total number } \\
\text { of words) }\end{array}$ & $\begin{array}{l}\text { Positive } \\
\text { words }\end{array}$ & $\begin{array}{l}\text { Negative } \\
\text { words }\end{array}$ & $\begin{array}{l}\text { Average } \\
\text { rating } \\
\text { Trendid }\end{array}$ \\
\hline 212 & 109 & 34 & 2.8266 Se \\
\hline 245 & 66 & 179 & $-2.1681 \mathrm{Ve}$ \\
\hline
\end{tabular}

\section{Word Cloud:}

Below figure representing non-stop words from a review file

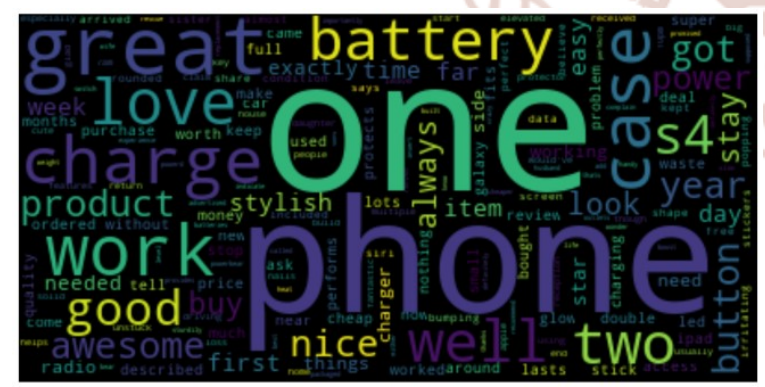

Figure 2: Word cloud representing nonstop-words

\section{CONCLUSION}

The goal is to give a feature based synopsis of an expansive number of client surveys of an item sold on the web. Our exploratory outcomes show that the proposed methods are extremely encouraging in playing out their assignments. We trust that this issue will turn out to be progressively essential as more individuals are purchasing and communicating their suppositions on the Web. Summarizing the reviews is not only useful to common shoppers, but also crucial to product manufacturers.

In our future work, we find a way to differentiate fake and genuine reviews with greater accuracy.

\section{REFERENCES}

1. JAWAD KHAN, BYEONG SOO JEONG , "SUMMARIZING CUSTOMER REVIEW BASED ON PRODUCT FEATURE AND OPINION", Proceedings of the 2016 International Conference on Machine Learning and Cybernetics, Jeju, South Korea, 10-13 July, 2016.

2. Gaurav Dubey, Ajay Rana, Naveen Kumar Shukla, "User Reviews Data Analysis using Opinion Mining on ", 2015 1st International Conference on Futuristic trend in Computational Analysis and Knowledge Management (ABLAZE-2015)

3. Priyanka C, Deepa Gupta, "Identifying the Best Feature Combination for Sentiment Analysis of Customer Reviews", Advances in Computing, Communications and Informatics (ICACCI), 2013 International Conference

4. LiZhen Liu, WenTao Wang, HangShi Wang, "Summarizing Customer Reviews Based On Product Features", Image and Signal Processing (CISP), 2012 5th International Congress

5. Minqing $\mathrm{Hu}$ and Bing Liu, "Mining and Summarizing Customer Reviews", Published in KDD, 2004

6. Santhosh Kumar K L, Jayanti Desai, Jharna Majumdar, "Opinion Mining and Sentiment Analysis on Online Customer Review", Computational Intelligence and Computing Research (ICCIC), 2016 IEEE International Conference

7. NeenaDevasia, Reshma Sheik, "Feature Extracted Sentiment Analysis of Customer Product Reviews", Emerging Technological Trends (ICETT), International Conference

8. ChhayaChauhan, SmritiSehgal, "SENTIMENT ANALYSIS ON PRODUCT REVIEWS", Computing, Communication and Automation (ICCCA), 2017 International Conference 
9. Shashank Kumar Chauhan, Anupam Goel, PrafullGoel, Avishkar Chauhan and Mahendra K Gurve, "Research on Product Review Analysis and Spam Review Detection", Signal Processing and Integrated Networks (SPIN), 2017 4th International Conference

10. A. Angelpreethi, Dr. S. BrittoRameshKumar, "AN ENHANCED ARCHITECTURE FOR FEATURE BASED OPINION MINING FROM PRODUCT REVIEWS", Computing and Communication Technologies (WCCCT), 2017 World Congress

11. Nilanshi Chauhan, Pardeep Singh, "Feature based Opinion Summarization of Online Product Reviews", Science Technology Engineering \& Management (ICONSTEM), 2017 Third International Conference

12. Costin-Gabriel, AsmelashTekaHadgu, "Sentiment Based Text Segmentation", Systems and Computer Science (ICSCS), 2013 2nd International Conference 